Please do not remove this page

RMIT

UNIVERSITY

\title{
Robust traffic engineering
}

Jennings, Andrew; Gregory, Mark; Agrawal, Himanshu

https://researchrepository.rmit.edu.au/esploro/outputs/9921864291401341/filesAndLinks?institution=61RMIT_INST\&index=null

Jennings, A., Gregory, M., \& Agrawal, H. (2008). Robust traffic engineering. Proceedings of the 2nd IEEE Symposium on Advanced Networks and Telecommunication Systems, 1-3.

https://doi.org/10.1109/ANTS.2008.4937808

Published Version: https://doi.org/10.1109/ANTS.2008.4937808

Repository homepage: https://researchrepository.rmit.edu.au

(c) 2008 IEEE. Personal use of this material is permitted. However, permission to reprint/republish this material for advertising or promotional purposes or for creating new collective works for resale or redistribution to servers or lists, or to reuse any copyrighted component of this work in other works must be obtained from the IEEE.

Downloaded On 2023/04/27 01:26:11 +1000 


\section{Robust Traffic Engineering}

\author{
Himanshu Agrawal \\ Electrical and Computer Engg. \\ RMIT University Australia \\ Email: himanshu.agrawal@rmit.edu.au.
}

\author{
Andrew Jennings \\ Electrical and Computer Engg. \\ RMIT University Australia \\ Email: ajennings@rmit.edu.au.
}

\author{
Mark Gregory \\ Electrical and Computer Engg. \\ RMIT University Australia \\ Email: mark.gregory@ rmit.edu.au.
}

\begin{abstract}
Phenomenal growth of Internet applications in recent years have made it difficult to forecast traffic patterns. Daily Internet traffic patterns shows that the network is vulnerable to malicious attacks, flash crowds and denial of service attacks (DDoS).

In this paper, we present a Robust Routing Technique (RRT) that attempts to deal with both normal routing conditions and transient failures. Our simulation results are compared with OSPF-TE. The key advantage of RRT is its convergence to generate the solution. It converges quickly to produce the simulation result on the family of topologies we consider in this paper. We are aiming to combine the best of proactive and reactive traffic engineering in RRT.
\end{abstract}

Keywords- Robust Routing, Traffic demand matrix, Traffic Engineering.

\section{INTRODUCTION}

Large IP networks are currently facing a challenge of routing under changing traffic conditions. Traffic Engineering (TE) is playing an important role in optimizing the traffic between ingress and egress (IE) node pairs. TE algorithms largely depend on the traffic pattern. The traffic pattern can be modeled as traffic demand. Traffic demand is normally stable most of the time, but there exists time interval when it is highly dynamic and unpredictable [2]. In recent years IP networks have experienced tremendous growth with services e.g. Voice over IP, Video on Demand, other multimedia streaming services etc. Hence traffic congestion and variability in traffic demand may affect IP carriers in service dimensions: travel time, reliability and cost. Therefore there is a need to develop robust routing tools that can accommodate the uncertainty in traffic demand data for Next Generation Internet. In this paper, we focus on uncertainty in traffic demand.

The actual distribution of traffic, entering at an ingress router to the various network egress routers, is not known and may vary over time. Intrinsic shift in traffic distribution may be caused by sudden appearance of flash-crowds responding to international events, denial-of service attacks, outbreak of worms and viruses. While local routing changes are under a carrier's control but unpredictable traffic shift can happen due to routing changes in other ASes.
Current methods to address the uncertainty in the TE problem follow the strategies:

- Prediction based .These algorithms are basically proactive to optimize the routing based on collected traffic samples. Proactive algorithms perform efficiently when traffic is stable but do not readjust to handle unpredictable traffic spikes.

- Reactive algorithms adapt to the traffic changes and converge quickly without making any prediction. Reactive approach is though responsive to the traffic changes, yet the issue of stability and convergence needs to be addressed both in theory and in practice.

We propose a solution by combining the proactive and reactive algorithms. In this paper, we consider the TE problem with demand uncertainty. Routing optimization problems are based on minimizing or maximizing objective function to optimize the network resources. A robust version of the minimization problem under uncertainty can be obtained by combined Minimization-Maximization. In our robust optimization methodology, we are interested in introducing demand uncertainty in a way to obtain a robust routing solution which can be solved efficiently. Applying a robust TE solution to normal traffic demand condition as well as dynamic traffic demand (due to DDoS or worms) may be a novel approach.

Contribution: The main contribution of this paper is to analyze our Robust TE algorithm (RRT) under uncertain traffic demand. We use an empirical simulation model to compare the RRT with OSPF. RRT may be static for multihour traffic. We are aiming to investigate RRT as simple, robust yet responsive to worms and DDoS. In this paper we propose RRT as simple and robust routing solution under dynamic traffic demand.

We believe that there is a need to do further studies of Robust TE that can give us better performance under dynamic traffic demand. Our research work is complementary to [4], and [7].

The remainder of the paper is organized as follows. We discuss the relevant literature in the next section II. In Section III, we present Robust TE problem and Linear Programming formulation In IV, we present simulation experiments and preliminary results. This includes a comparison of the robust solution on family of topologies showing that robust routing solution better addresses traffic demand uncertainty. We conclude our paper with future work in Section V. 


\section{RELATED WORK}

In the recent years robust TE has attracted significant attention of the network and operation research community. TE algorithms can be broadly classified as proactive (prediction based) and reactive to the unpredictable changes in the traffic demand.

Proactive TE: One of the earliest and benchmark research papers in this category was proposed by Applegate and Cohen [1]. The authors use a limited knowledge of traffic demand to obtain an efficient routing called oblivious routing. Furthermore, they provide a lower bound on the performance for the routing under all possible traffic situations. Fortz and Thorup [4] use a search heuristics for OSPF/IS-IS in finding suitable link weight settings to a given traffic situations. Azar et al [3] have shown that the routing performance metric is relative and it does not give any guarantee about the absolute performance of the selected routing. Wang et al. propose a deterministic proactive approach using a notion of penalty envelope and modeling traffic demand as a convex-hull in their paper COPE [9].

Reactive TE: More recently, there have been proposals for reactive or online Multipath TE [6,7]. A distributed method called TeXCP for MPLS traffic engineering was introduced by Khandula et al. [7]. Load balancing is performed over a set of precomputed MPLS paths between ingress and egress routers based on traffic measurement from the network. The authors discussed and prove stability, convergence and optimality as well. Reactive routing presents a desirable property of keeping routing adapted to dynamic traffic. However, reactive routing algorithms show poor performance under abrupt traffic changes [9].

We extend the previous solution approach COPE [9] by combining proactive and reactive TE. We study the robust routing under uncertain traffic demand in our paper [8].

\section{ROBUST TRAFFIC ENGINEERING}

We consider a directed graph $\mathrm{G}=(\mathrm{N}, \mathrm{A})$ where $\mathrm{N}$ is the set of nodes and $\mathrm{A}$ is set of arcs. The graph represents the backbone network and arcs are referred to as the physical and logical connections between two nodes in the network. Input to the TE is the traffic matrix(TM). Output of the TE is the routing with the set of feasible robust routing paths.

We define traffic demand polytope $D$ as the uncertainty set to map the dynamic nature of internet traffic. From Minkowski's theorem a traffic demand polytope $D$ is the convex hull of its extreme points, i.e.

$D=\left\{t \in \mathrm{R}_{+}|\mathrm{N}|(|\mathrm{N}|-1): t=\left(\mathrm{d}_{\mathrm{ij}}\right)_{\mathrm{i}, \mathrm{j} \in \mathrm{N}}\right\}$

This $\left(\mathrm{d}_{\mathrm{ij}}\right)_{\mathrm{i}, \mathrm{j}} \in \mathrm{N}$ defines a traffic demand vector between all IE pairs of nodes of $\mathrm{N}$ and this vector will be known as traffic matrix. This traffic matrix may be a variable or can be a point of traffic demand polytope $D$.

We formulate the problem as multicommodity arc-path flow problem with an objective function as Maximum Link Utilization subjected to set of constraints. We denote each entry of traffic profile as a traffic demand between an IE pair. We introduce the following notation to formulating the problem: $\mathrm{d}_{\mathrm{ij}}$ : Traffic demand between node ingress $\mathrm{i}$ and each egress node $\mathrm{j},(\mathrm{i}, \mathrm{j} \in N)$

$\mathrm{r}_{\mathrm{ij}}{ }^{\mathrm{p}}$ : Fraction of traffic demand from $\mathrm{i}$ to $\mathrm{j}$ carried through the path $p \in P(i, j)$

$\mathrm{r}_{\mathrm{ij}}{ }^{\mathrm{a}}$ : Fraction of traffic demand from $\mathrm{i}$ to $\mathrm{j}$ carried through arc $\mathrm{a} \in \mathrm{A}$.

$c_{a}: \quad$ Capacity of each arc a.

maxU : Maximum Link Utilization

$\mathrm{h}_{\mathrm{a}}: \quad$ volume of traffic on each $\operatorname{arc}=\sum_{i, j} \mathrm{~d}_{\mathrm{ij}} \mathrm{r}_{\mathrm{ij}}{ }^{\mathrm{a}}$

Minimize $\max U$

$$
\begin{aligned}
& \text { Subject to: } \\
& \sum_{p \in P(i, j)} \mathrm{r}_{\mathrm{ij}}^{\mathrm{p}} \geq 1 \quad \forall \mathrm{i}, \mathrm{j} \in \mathrm{N} \\
& \sum_{p \in P(i, j)} \mathrm{r}_{\mathrm{ij}}^{\mathrm{p}} \leq \mathrm{r}_{\mathrm{ij}}{ }^{\mathrm{a}} \quad \forall \mathrm{i}, \mathrm{j} \in \mathrm{N}, \forall \mathrm{a} \in \mathrm{A} \\
& \sum_{i, j \in N} \mathrm{~d}_{\mathrm{ij}} \mathrm{r}_{\mathrm{ij}}^{\mathrm{a}} \leq \operatorname{maxU} \cdot \mathrm{c}_{\mathrm{a}} \quad \forall \mathrm{d}_{\mathrm{ij}} \in D \\
& \mathrm{r}_{\mathrm{ij}}{ }^{\mathrm{p}} \geq 0 \quad \forall \mathrm{p} \in \mathrm{P}(\mathrm{i}, \mathrm{j}), \quad \forall \mathrm{i}, \mathrm{j} \in \mathrm{N} \\
& \mathrm{r}_{\mathrm{ij}}{ }^{\mathrm{a}} \geq 0 \quad \forall \mathrm{a} \in \mathrm{A}, \quad \forall \mathrm{i}, \mathrm{j} \in \mathrm{N}
\end{aligned}
$$

This formulation is based on arc-path flow formulation. The first constraint in inequality (3) refers to the Multipath routing as traffic demand between each pair of nodes may split among multiple paths. Flow variable for each is defined by an inequality (4). Total traffic flowing through each arc must be less than the capacity times the MLU, defined by constraint in (5).Given the traffic demand matrix and weights the objective is to minimize the maxU or $\max \left(\mathrm{h}_{\mathrm{a}} /\right.$ $c_{a}$ ), that will result in minimum worst case utilization over all the arcs in the network. The problem of routing traffic demands to minimize congestion over multiple paths is NPhard [5]. Thus we resort to local search heuristic proposed by Fortz and Thorup in [4].

Routing Optimization refers to finding set of paths between each pair of IE routers by optimizing an objective function subjected to traffic demand and capacity constraints. Our basic approach is shown (see figure 1), in which a traffic demand with network topology forms the input to a routing master program that generates a set of robust and optimal paths. The framework of routing algorithm is as follows:

\section{INPUT: Set of nodes N, Set of arcs A, traffic Demand OUTPUT: Set of robust paths \\ Step1: Initialize with intradomain topology. \\ Step2: Compute the LP of the equations in (2). \\ Step3: Path decomposition to compute Set of Paths. \\ Step4: Path Update}

We use Dijkstra's algorithm to compute the initial path set. In the next step, we formulate LP using equations (2) to (7). Using the LP to solve the problem at each step, we find the paths with minimized Maximum Link Utilization. We update these paths to the active path set; in step 3 and 4 . We are using column generation approach. This column generation procedure will only compute and add the subset 
of paths that can reduce the value of Maximum Link Utilization at each step. A master program solving each sub problem based the constraint will prove optimality of current solution. Our goal is to focus on a hybrid routing solution to bridge the gap between reactive and proactive TE methods.

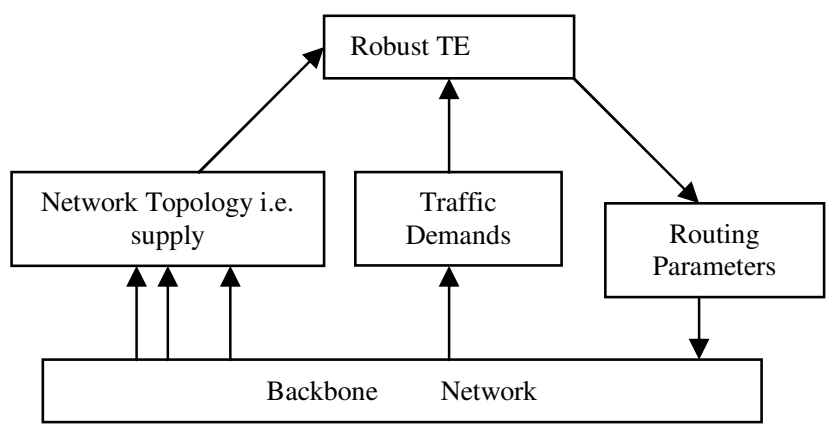

Figure 1. Robust TE Schematic

\section{RESULTS AND DISCUSSION}

We use following techniques for simulation:

OSPF Optimizer: We use OSPFOpt [7] for our preliminary experiments to evaluate the network performance. Given a traffic demand matrix and topology OSPF- TE computes a set of weights which when used with OSPF protocol result in low cost or low Maximum Link Utilization.

\begin{tabular}{|l|c|c|c|c|}
\hline \multirow{2}{*}{\multicolumn{1}{|c|}{ ISP(\#AS) }} & \multirow{2}{*}{$\mathrm{N}, \mathrm{A}$} & \multicolumn{3}{|c|}{ Max-Utilization } \\
\cline { 3 - 5 } & & OSPF & $\begin{array}{c}\text { RRT } \\
\text { (ECMP) }\end{array}$ & $\begin{array}{c}\text { RRT } \\
\text { (WSP) }\end{array}$ \\
\hline Telstra (1221) & 7,18 & 0.612 & 0.5703 & 0.570 \\
Sprint (1239) & 27,126 & 0.863 & 0.6934 & 0.703 \\
Abovenet & 17,74 & 0.370 & 0.4673 & 0.467 \\
(6461) & 18,66 & 0.375 & 0.4236 & 0.423 \\
Ebone( 1755) & & & & \\
\hline
\end{tabular}

TABLE I. Rocketfuel [2] topologies and performance evaluation (Traffic Margin $=1.1$ )

\begin{tabular}{|c|c|c|c|c|}
\hline \multirow[b]{2}{*}{ ISP(\#AS) } & \multirow[b]{2}{*}{$\mathrm{N}, \mathrm{A}$} & \multicolumn{3}{|c|}{ Max-Utilization } \\
\hline & & OSPF & $\begin{array}{r}\text { RRT } \\
(\mathrm{ECMP})\end{array}$ & $\begin{array}{r}\text { RRT } \\
\text { (WSP) }\end{array}$ \\
\hline Telstra (1221) & 7,18 & 0.652 & 0.652 & 0.6525 \\
\hline Sprint (1239) & 27,126 & 0.719 & 0.719 & 0.7193 \\
\hline $\begin{array}{l}\text { Abovenet } \\
\text { (6461) }\end{array}$ & 17,74 & 0.335 & 0.585 & 0.5851 \\
\hline Ebone( 1755) & 18,66 & 0.366 & 0.426 & 0.4268 \\
\hline
\end{tabular}

TABLE II. Rocketfuel topologies and performance evaluation $($ Traffic Margin $=3.0)$

RRT: We use Stanford Graph Base software (SGB) [5] to simulate the Robust TE module shown in the Figure-1 to provide sets of paths corresponding to computed link weights and traffic traces under changing traffic demand conditions. Weighted Shortest Path (WSP) and Equal Cost
Multi-Paths (ECMP) algorithms are both implemented in RRT for traffic allocation. Maximum Link Utilization is calculated based on both OSPF and RRT.

RRT under uncertain traffic demands- Our simulation results demonstrate that RRT performs better on topologies 1221 and 1239 compare to OSPF-TE. We performed several simulation runs by providing range of traffic matrices to OSPF and RRT. Moreover two different Traffic Margins are provided to the traffic matrix as shown in the Table-I and Table-II to test the robustness. Traffic Margins are provided to the base traffic demand where each entry in the TM increases or decreases by small amount according to the equation (1). It is evident from the data in the above tables that RRT offers reasonable Max-Utilization in some cases but may suffer for some topologies. We run our simulation on Intel Core 2 Duo processor, 1 GB RAM and Windows XP. OSPF Optimizer takes 5000 iterations before generating the solution. This may result in a considerable time even for a topology with 50 nodes. On the other hand RRT which is using SGB [5] takes few seconds for all the topologies, we use in our simulation.

\section{CONCLUSION AND FUTURE WORK}

Our preliminary results demonstrate the performance of RRT compared to OSPF-TE. We are currently testing RRT on different demand polytope and investing further to see the feasibility of RRT as robust, stable yet reactive to DDoS or worms. It is also interesting to test RRT for effect of interdomain traffic demand changes.

\section{ACKNOWLEDGMENT}

We are thankful to Shrikanth Kandula for providing us the modified Rocketfuel topologies. We thank Duc Quang for helping in coding RRT. We are also thankful to all the reviewers for their valuable comments.

\section{REFERENCES}

[1] D. Applegate, E. Cohen, Making intra-domain routing robust to changing and uncertain traffic demands: understanding fundamental tradeoffs. Proceeding of SIGCOMM'03, Karlsruhe, Germany, pp. 313-324.

[2] N. Spring, R. Mahajan and D. Wetherall, Measuring ISP topologies with Rocketfuel. In proceedings of the ACM SIGCOMM Conference. 2002.

[3] Y. Azar, E. Cohen, A. Flat, H. Kalplan and H. Racke. Optimal oblivious routing in polynomial time. In Proceedings of the $35^{\text {th }} A C M$ Symposium on the Theory of Computing, 2003.

[4] B. Fortz. And M. Thorup. Optimizing OSPF/IS-IS weights in a changing world. IEEE journal on selected areas in communications, 20(4), 2002.

[5] D. E. Knuth, The Stanford GraphBase: A Platform for Combinatorial Computing. New York: ACM Press, 1993.

[6] A. Elwalid, C.Jin, S.Low and I. Widjaja. MATE: MPLS adaptive traffic engineering. In proceedings of INFOCOMM'01 page 1300-1309, Anchorage, April 2001.

[7] S. Kandula, D. Katabhi, B. Davie and A. Charny. Walking the tightrope: Responsive yet stable traffic engineering. In proceedings of SIGCOMM' 05 pages 253-264, Philadelphia, August 2005.

[8] H. Agarwal, A. Jennings, Mark Gregory and Daud Channa Robust Routing, In the proceeding of Seventh IEEE/ACIS International conference, Portland, Oregon, May 2008.

[9] H. Wang, H. Xie, L. Qiu, Y.R. Yang, Y. Zhang and A. Greenberg. COPE: Traffic engineering in dynamic networks. In SIGCOMM'06. 